\title{
Prophylactic Topical Tranexamic Acid Versus Placebo in Surgical Patients
}

\author{
A Systematic Review and Meta-Analysis*
}

\author{
Wan Yi Teoh, MBChB, ${ }^{*}$ Tun Giap Tan, BMBS, † Ka Ting Ng, MBChB, $\dagger$ Ke Xin Ong, $\S$ \\ Xue Lin Chan, MBChB, $\ddagger$ Samuel Ern Hung Tsan, BMBS, $\uparrow$ \\ and Chew Yin Wang, MBChB, FRCA, FFARCS $\ddagger$
}

\begin{abstract}
Objectives: Perioperative bleeding remains a major concern to all clinicians caring for perioperative patients. Due to the theoretical risk of thromboembolic events associated with tranexamic acid (TXA) when administered intravenously, topical route of TXA has been extensively studied, but its safety and efficacy profile remain unclear in the literature. The primary aim of this review was to assess the effect of topical TXA on incidence of blood transfusion and mortality in adults undergoing surgery.

Data sources: EMBASE, MEDLINE, CENTRAL, and ISI Web of Science were systematically searched from their inception until May 31, 2019.

Review methods: Parallel-arm randomized controlled trials were included. Results: Seventy-one trials (7539 participants: orthopedics 5450 vs nonorthopedics 1909) were included for quantitative meta-analysis. In comparison to placebo, topical TXA significantly reduced intraoperative blood loss [mean difference (MD) $-36.83 \mathrm{~mL}, 95 \%$ confidence interval (CI) -54.77 to $-18.88, P<0.001]$, total blood loss (MD $-319.55 \mathrm{~mL}, 95 \% \mathrm{CI}-387.42$ to $-251.69, P<0.001$ ), and incidence of blood transfusion [odds ratio (OR) $0.30,95 \%$ CI $0.26-0.34, P<0.001]$. Patients who received topical TXA were associated with a shorter length of hospital stay (MD -0.28 days, $95 \% \mathrm{CI}$ -0.47 to $-0.08, P=0.006)$. No adverse events associated with the use of topical TXA were observed, namely mortality (OR $0.78,95 \%$ CI $0.45-1.36, P$ $=0.39$ ), pulmonary embolism $(\mathrm{OR} 0.73,95 \%$ CI $0.27-1.93, P=0.52)$, deep vein thrombosis (OR 1.07, 95\% CI 0.65-1.77, $P=0.79$ ), myocardial infarction (OR $0.79,95 \%$ CI $0.21-2.99, P=0.73$ ), and stroke (OR 0.85 , $95 \%$ CI $0.28-2.57, P=0.77)$. Of all included studies, the risk of bias
\end{abstract}

From the *University of Liverpool, School of Medicine, Cedar House, Liverpool, United Kingdom; †Basingstoke and North Hampshire Hospital, Hampshire, United Kingdom; $\ddagger$ Department of Anaesthesiology, Faculty of Medicine, University of Malaya, Jalan University, Kuala Lumpur, Malaysia; §University of Warwick, Gibbet Hill Road, Coventry, United Kingdom; and -Faculty of Medicine and Health Sciences, University of Malaysia Sarawak, Sarawak, Malaysia.

凶katingng1@gmail.com.

ORCID ID: 0000-0001-7825-2628

* The abstract was accepted for an oral presentation at the Association of Surgeons in Training (ASiT) conference in Birmingham (6-8 March 2020).

The authors report no conflicts of interest.

This review was registered in PROSPERO (CRD 42018111762).

Financial support and sponsorship: This research did not receive any specific grant from funding agencies in the public, commercial, or not-for-profit sectors.

Supplemental digital content is available for this article. Direct URL citations appear in the printed text and are provided in the HTML and PDF versions of this article on the journal's Web site (www.annalsofsurgery.com).

Authors' contributions: W.T.: protocol/ project management, data collection or management, data analysis, manuscript writing/editing; T.T.: protocol/ project management, data collection or management;K.N.: protocol/ project management, data collection or management, data analysis, manuscript writing/ editing; K.O.: protocol/ project management, data collection or management; X.C.: protocol/ project management, data collection or management; S.T. protocol/ project management, data collection or management; C.W.: protocol/ project management, data analysis, manuscript writing/editing.

Copyright (C) 2020 Wolters Kluwer Health, Inc. All rights reserved.

ISSN: 0003-4932/16/XXXX-0001

DOI: $10.1097 /$ SLA.0000000000003896 assessment was "low" for 20 studies, "unclear" for 26 studies and "high" for 25 studies.

Conclusions: In the meta-analysis of 71 trials (7539 patients), topical TXA reduced the incidence of blood transfusion without any notable adverse events associated with TXA in adults undergoing surgery.

PROSPERO: CRD 42018111762.

Keywords: meta-analysis, mortality, systematic review, topical agents, tranexamic acid, transfusion

(Ann Surg 2020;xx:xxx-xxx)

atients undergoing major surgery are prone to substantial intraoperative and postoperative blood loss. ${ }^{1-4}$ Although allogenic blood transfusion is a life-saving measure, it may lead to serious adverse events, namely hemolytic transfusion reaction, transfusionrelated acute lung injury, and transmission of viral or bacterial infections. ${ }^{5,6}$ All these adverse events could prolong the duration of hospital stay, increase cost of hospitalisation, reduce quality of life, and, at worst, may increase the risk of mortality. ${ }^{7}$

Many alternative strategies are available to reduce blood loss perioperatively, namely use of tourniquet, ${ }^{8}$ hypotensive anesthesia, ${ }^{9}$ fibrin sealant, ${ }^{10}$ and topical pharmacological agents. ${ }^{11}$ Of these, prophylactic administration of tranexamic acid (TXA) before or during the major surgery is believed to reduce blood loss and incidence of blood transfusion. TXA is an anti-fibrinolytic agent approved for preventing or treating bleeding in different clinical settings. It is a highly efficacious synthetic amino acid lysine analogue, ${ }^{12,13}$ and is deemed as a cost-effective drug. ${ }^{14}$ TXA competitively binds to the lysine-binding site of plasminogen to inhibit the activation of plasminogen to plasmin and prevent the degradation of fibrin clot, resulting in delaying fibrinolysis, enhancing hemostasis and reducing blood loss. ${ }^{12,13}$ Given its anti-fibrinolytic mechanism of action, thrombotic complications are a potential risk.

In light of the safety concern regarding the theoretical risk of fatal thromboembolic event with intravenous administration of TXA, ${ }^{15}$ topical route of TXA has been proposed as an alternative measure to minimize blood loss. The plasma concentration of topical TXA was found to be $90 \%$ lower than intravenous TXA. ${ }^{16}$ However, topical TXA has been shown to result in a higher therapeutic concentration at the bleeding site with minimal or no systemic absorption. ${ }^{16,17}$ Many recent trials investigating the benefits of topical TXA in surgery have been published with conflicting results. ${ }^{18-23}$ Consequently, the efficacy and safety profile of topical TXA remains unclear in the literature. A systematic review and metaanalysis are timely warranted to synthesize current evidence on the prophylactic use of TXA in adults undergoing surgery.

The primary aim of this systematic review and meta-analysis was to evaluate the effects of topical TXA on incidence of blood transfusion and mortality in adults undergoing surgery. Secondary aims were to investigate the clinical effects of TXA on bleeding 